\title{
Preparation and properties of silk sericin/cellulose cross-linking films
}

\author{
Kunyan Wang ${ }^{\mathrm{a}}$, and Fangyu Zhan \\ Department of Materials Chemistry, Huzhou University, zhuzhou, 313000, China
}

\begin{abstract}
Silk sericin/cellulose cross-linked films were successfully prepared using glutaraldehyde as crosslinkinger. FTIR was applied to characterize the chemical structure of films. Cross-linked silk sericin film was found the peak intensity of FTIR for cross-linked film decreased markedly compared to pure silk sericin, which indicating cross-linking reaction has been occurred. The increasing value of swelling ratio also indicated the cross-linking has been happened. The cross-linking reaction increased the thermal decomposition temperature.
\end{abstract}

\section{Introduction}

Biomaterials such as silk sericin and cellulose are considered as potential substitutes for synthetic polymers. Silk sericin delivered from silkworm silk, has been explored as a versatile biomaterial for various fields. It has excellent mechanical properties with high elastic modulus, elongation to break, and toughness [1-3]. In recent years biomaterials using flexible electronics based on organic field-effect transistors (OFETs) have attracted considerable attention. It have been introduced as gate dielectrics with low dielectric losses and leakage currents as well as sufficiently high dielectric breakdown strength. It has been introduced as gate dielectrics. B. Singh et. al. [4] fabricated an OFET device using a deoxyrionucleiv acid-based biopolymer for gate dielectric region. J.W. Chang et. al. [5] used albumen, as a gate dielectric in pentacene- and $\mathrm{C}_{60}$-based organic field-effect transistors.

However, Silk sericin materials are either too weak in wet state or too brittle and stiff in the dry state to application. Blending is an effective possible way to combine advantanges of each componet[6]. Blending of silk sericin and cellulsoe have been prepare to acquire biomaterial that are able to obtain good mechanical properites[7]. Meanwhile, to maintain water-insolubility chemical crosslinking reagents were added to blending film. Glutaraldehyde is an effective synthetic crosslinking agents that can react with amino acids or proteins [8]. It has been reported biopolymers using glutaradehyde as crosslinking agents show good mechnical properites.

In this work, silk sericin and cellulose cross-linking films were prepared. The effects of diffenernt cellulose content on the chemical structure of the silk sericin and the thermal behavior were confirmed by FTIR and DSC.

\section{Experimental Section}

\subsection{Materials}

Silk sericin powder was purchased from Huzhou Xintiansi Biotec Co. (China). Glutaraldehyde was supplied by LingFeng Chemical Reagent Co. Ltd.. Cellulose was purchased from Aladdin Industrial Corporation (China). All chemical were of analytical grade, and used as received without further purification.

\subsection{Specimens preparation}

First $8 \%$ carbamide, $8 \%$ sodium hydrate and $6.5 \%$ thiocarbamide were mixed into conical flask with continuous magnetic stirring to make mixed solution. Silk sericin and cellouse with $2 \%$ contents of glutaraldehydewere dissolved into above mixed solution for $2 \mathrm{~h}$ to generate a homogeneous solution. Finally the homogenous solution was poured into a smooth plate and placed in an oven at $60^{\circ} \mathrm{C}$ until dried silk sericin/cellouse samples were obtained.

\subsection{Characterizations}

Fourier-transform infra-red (FTIR) spectroscopy has been used to monitor the chemical reactions. FTIR were recorded by nicolet NEXUS-670 IR spectrometer in the wavelength range of $4000-400 \mathrm{~cm}^{-1}$.

$\mathrm{X}$-ray diffraction patterns were recorded in XD-6 Xray diffractometer (China). The length and width of all test samples are $20 \mathrm{~mm}$ and $15 \mathrm{~mm}$, respectively. The wavelength of the monochromated X-ray from $\mathrm{Cu} \mathrm{Ka}$ radiation is $0.154056 \mathrm{~nm}$. Scanning $2 \theta$ range was $10-60^{\circ}$ with a scanning rate of $2 \% \mathrm{~min}$.

Differential scanning calorimetry was used to measure the melting temperature of samples. Sample weights ranged from 3 to $5 \mathrm{mg}$. All samples were heated

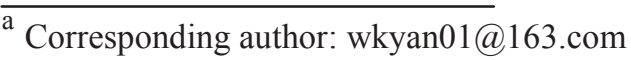


from room temperature to $400^{\circ} \mathrm{C}$ at a rate of $20^{\circ} \mathrm{C} / \mathrm{min}$. Heating curves was recorded.

The weight of $0.1 \mathrm{~g}$ dry sample was immersed into adequate deionizer water at room temperature for $24 \mathrm{~h}$ to reach the swelling equilibrium. Then, the swelling ratio $(\mathrm{g} / \mathrm{g})$ of sample was calculated.

\section{Results and discussion}

\subsection{Chemical structure of silk sericin/cellouse cross-linked films}

The FTIR spectra of pure silk sericin, pure cellouse and silk sericin/cellouse cross-linked films with $2 \%$ glutaraldehyde were shown in Fig.1. Silk sericin exhibited characterisitc amide absorption bands of protein, amide, and at 1635,1415 , and $1093 \mathrm{~cm}^{-1}$, respectively, which indicating random coil structures of silk sericin $[6$, 7]. Cellouse exhibited characterisitc bands at 1635, 1419, 1310 and $1084 \mathrm{~cm}^{-1}$, respectively. The peak at $1635 \mathrm{~cm}^{-1}$ was mainly corresponded with the stretching vibrations of $\mathrm{C}=\mathrm{O}$ and $-\mathrm{NH}$ group. Compared to pure silk sericin and cellouse, cross-linked film with $2 \%$ glutaraldehyde was found the peak intensity decreased markedlly, which indicating crosslinking reaction has been occurred.

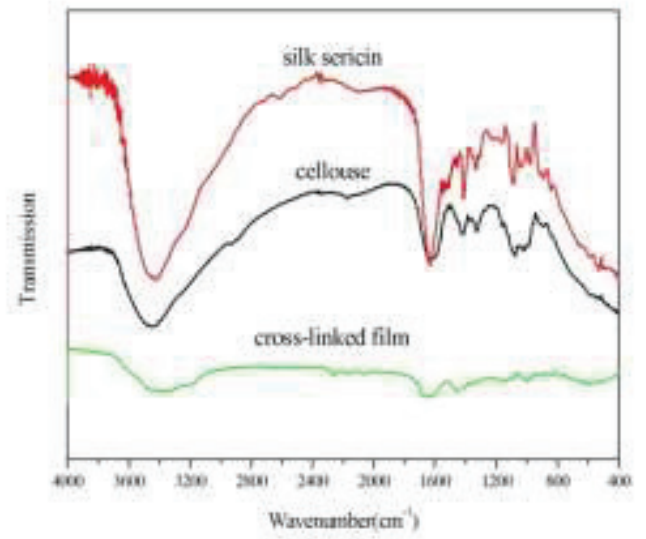

Figure 1. Infrared spectrums of pure silk sericin, pure cellouse and cross-linked film with $2 \%$ glutaraldehyde.

\subsection{Crystal structure of silk sericin/cellouse cross-linked films}

Fig. 2 shows the XRD patterns of pure silk sericin, pure cellouse and $2 \%$ glutaraldehyde cross-linked silk sericin films. Diffraction peaks of $2 \theta=21.9^{\circ}, 28.0^{\circ}$ for pure silk sericin are assigned to the silk II crystal planes, which is the spun silk state ( $\beta$-sheet silk structure). Pure cellouse shows diffraction peaks at $2 \theta=22^{\circ}, 34.6^{\circ}$. For $2 \%$ glutaraldehyde cross-linked film, it can be found that the diffraction peaks of $2 \theta=21.9^{\circ}$ disappears. The results show that the silk sericin crystalline structure affected by glutaraldehyde and cellouse.

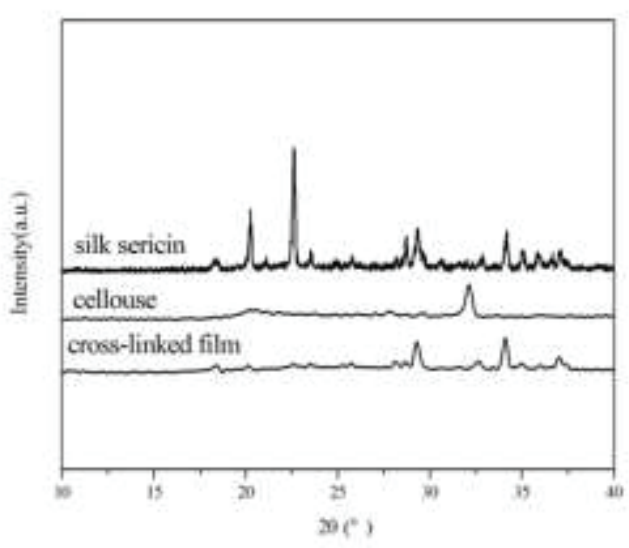

Figure 2. XRD patterns of of pure silk sericin, pure cellouse and cross-linked film with $2 \%$ glutaraldehyde.

\subsection{Thermal behavior of silk sericin/cellouse cross-linked films}

Fig. 3 shows the DSC thermograms of heating scan for pure silk sericin, pure cellouse and cross-linked film with $2 \%$ glutaraldehyde from room temperature to $400{ }^{\circ} \mathrm{C}$. Both silk sericin and cross-linked film display a peak nearly $150{ }^{\circ} \mathrm{C}$, which is caused by desorption of water and motivation of molecular chain in amorphous region $[8,9]$. Meanwhile, an weak endothermic peak at $300{ }^{\circ} \mathrm{C}$ have appeared for pure silk sericin, representing the thermal decomposition of silk sericin. And then, an interse endothermic peak at $300{ }^{\circ} \mathrm{C}$ comes out in cellouse, representing the thermal decomposition of cellouse [10]. The peak at $300{ }^{\circ} \mathrm{C}$ disappeared for cross-linked film compared to pure silk sericin, which indicating the crosslinking reaction increasing the thermal decomposition temperature.

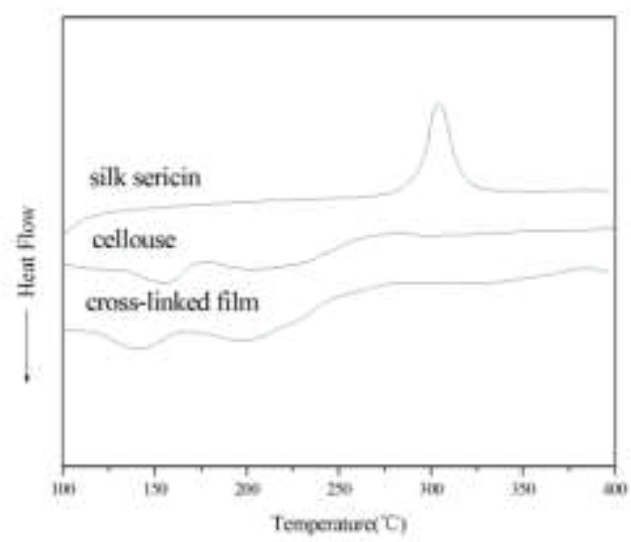

Figure 3. DSC thermograms of the second heating scan of pure silk sericin, pure cellouse and cross-linked film with $2 \%$ glutaraldehyde.

\subsection{Swelling behaviors of silk sericin/cellouse cross-linked films}

The swelling capacity in deionized water has been studied to evaluate the water-holding capacity. The 
swelling capacities of pure silk sericin and cross-linked silk sericin film were shown in Fig. 4. The swelling ratio of cross-linked silk sericin film increased compared to pure silk sericin. Swelling behavior depends on hydrophilicity/hydrophobicity, crosslinking density and the characteristics of the external solution. The increasing value of swelling ratio indicated the crosslinking have been happened.

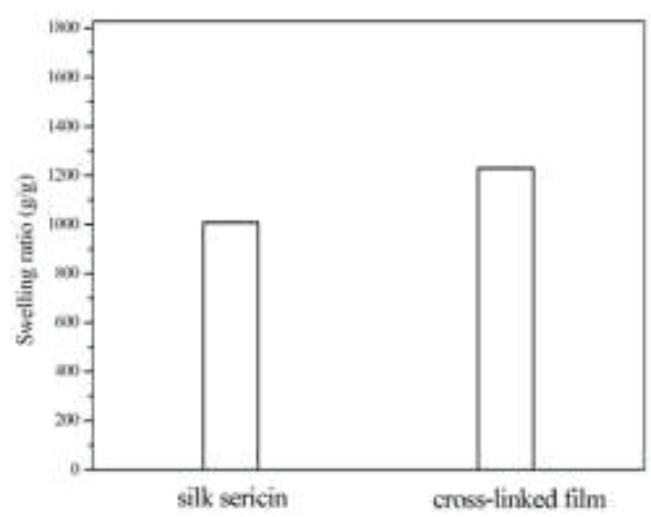

Figure 4. Swelling ratios of pure sericin and cross-linked film.

\section{Conclusions}

In this work, we report silk sericin/cellouse cross-linked films based on glutaraldehyde as crosslinking agent. FTIR was used to confirm the cross-linking structure of cross-linked films. The cross-linking reaction increased the thermal decomposition temperature. The increasing value of swelling ratio indicated the crosslinking have been happened.

\section{Acknowledgements}

The authors would like to acknowledge financial support by the Natural Science Foundation of Zhejing province of China (LQ14B040003).

\section{References}

1. Y.N. Lin, X.X. Xia, K. Shang, R. Elia, W.W. huang, P. Cebe, G. Leisk, F. Omenetto, D.L. Kaplan, Biomacromolecules. 14, 2629 (2013)

2. Y.P. Huang, B.P. Zhang, G.W. Xu, W.T. Hao, Composite. Sci. and Tech. 84, 15 (2013)

3. J. Luo, L. Zhao, Y.Y. Yang, G.L. Song, Y. Liu, L.J. Chen, G.Y. Tang, Solar Energy Mater. And Solar Cells. 147, 144 (2016)

4. B. Singh, N.S. Sariciftci, J. Appl. Phys. 100, 024514 (2006)

5. J.W. Chang, C.G. Wang, C.Y. Huang, T.D. Tsai, T.F. Guo, T.C. Wen, Adv. Mater. 23, 4077 (2011)

6. T.D. Thanh, L.Kapralkova, J. Hromadkova, I. Kelnar, Eur. Polym. J. 50, 39 (2014)

7. L. Zhou, Q. Wang, J.C. Wen, X. Chen, Z.Z. Shao, Polymer. 53, 5035 (2013)

8. A. Vasconcelos, A.C. Gomes, A. Cacaco-paulo, Acta.
Biomater. 8, 3049 (2012)

9. E. S. Sashina, G. Janowska, M. Zaborski, A.V. Vnuchkin, J. Therm. Anl. Calorim. 89, 887 (2007)

10. L. Zhou, Q. Wang, J. C. Wen, X. Chen, Z. Z. Shao, Polymer. 54, 5035 (2013) 